\title{
Using Fuzzy c-Means for Weighting Different Fuzzy Cognitive Maps
}

\author{
Mamoon Obiedat ${ }^{1}$ \\ Computer Information Systems Department \\ Hashemite University \\ Zarqa, Jordan
}

\author{
Ali Al-yousef ${ }^{2}$ \\ Computer Science Department \\ Jerash University \\ Jarash, Jordan
}

\author{
Ahmad Khasawneh ${ }^{3}$ \\ Prisednt of Irbid National University \\ Irbid National University \\ Irbid, Jordan
}

\author{
Nabhan Hamadneh $^{4}$, Ashraf Aljammal ${ }^{5}$ \\ Computer Science Department \\ Hashemite University \\ Zarqa, Jordan
}

\begin{abstract}
Currently, complex socio-ecological problems have increasingly prevailed with uncertainty that often dominates these domains. In order to better represent these problems, there is an urgent need to engage a wide range of different stakeholders' perspectives, regardless of their levels of expertise and knowledge. Then, these perspectives should be combined in an appropriate manner for a comprehensive and reasonable problem representation. Fuzzy cognitive map (FCM) has proven to be powerful and useful as a soft computing approach in addressing and representing such problem domains. By the FCM approach, the relevant stakeholders can represent their perspectives in the form of FCM system. Normally, relevant stakeholders have different levels of knowledge, and hence produce different representations (FCMs). Therefore, these FCMs should be weighted appropriately before the combination process. This paper uses fuzzy c-means clustering technique to assign different weights for different FCMs according to their importance in representing the problem. First, fuzzy c-means is used to compute the membership values of belonging of FCMs to the selected clusters based on the FCMs similarities that show how convergent and consistent they are. According to these membership values, the importance clusters' values are calculated, in which a cluster with a high membership value from all FCMs is the cluster with the high importance value, and vice versa. Next, the importance values for FCMs are derived from the importance values of the clusters by looking at the amount of contributions of FCMs memberships to the clusters. Finally, FCMs importance values are used to assign weight values to these FCMs, which are used when they are combined. The suitability of the proposed method is investigated using a real dataset that includes an appropriate number of FCMs collected from different stakeholders.
\end{abstract}

Keywords-Complex problems; uncertainty; fuzzy cognitive map (FCM); fuzzy c-Means; FCM weight values

\section{INTRODUCTION}

The world is facing a large number of various real problems in all aspects of life. On one hand, real-world problems have become complex in nature and are usually multidisciplinary. On the other hand, solving these problems has become an urgent necessity and attracted the attention of decision makers. However, modelling and capturing the knowledge of these problems face several key challenges, such as a lack of structural/quantitative data, domain complexity, and a lack of sufficient data (comprehensive view) representing adequately the domain knowledge. Moreover, such problems are often characterized by uncertainty, ill-defined, and qualitative imprecise data [1]. In such a case, hard computing approaches may not provide problem modelling or solving [2]. These problems can also include multiple dimensions, and hence, this leads to increase their complexity. For example, environmental problems usually include social/human and ecological dimensions [3]. This highlights the need to share the knowledge of all these dimensions. Here, a comprehensive view of such problem domains can embrace complexity, yet it requires an understanding of their dimensions and components on one side, and the players who interact between these dimensions and components on the other side [4-9].

In fact, soft computing approaches can deal with complex unstructured real-world problems including uncertainty and imprecise data with partial truth [2]. One helpful soft computing approaches that appropriately addresses uncertainty usually inherited in the real-world problems is a Fuzzy Cognitive Map (FCM). FCM approach incorporates the cognitive map concept with the fuzzy logic concept for expressively representing the knowledge of the problem. The purpose of the cognitive map is to represent the domain knowledge as map nodes representing the domain variables and directed arrows representing the connections between these variables [10]. On the other side, fuzzy logic has tolerance in modelling such problems and provides reasoning decision making [11]. It also can mathematically represent the imprecise data in a degree of truth (i.e. degree of membership in the range $[0,1])[12]$. FCM can address the multiple dimensionality of the problem domains including different related perspectives. It represents any complex problem in the form of variables and negative/positive connections between them $[1,7,13]$. The values of connections can be in the form of fuzzy numerical values (i.e. $[-1,1]$ ) or fuzzy linguistic values (i.e. weak, moderate, strong etc.). Moreover, FCM is easily converted to 
an adjacency matrix containing the connection values for mathematically handling.

To overcome the challenges of insufficient data and inherited uncertainty of large complex problems, several studies have suggested engaging a large sample of relevant stakeholders in representing the domain knowledge and capturing their perspectives/FCMs [1, 14-17]. Gathering many perspectives would lead to explore many relevant variables and connections with more certainty. Then, the perspectives of the stakeholders are combined to form a comprehensive perspective/FCM with more reliability and relevance in knowledge representation and problem solving [7, 14, 18]. In fact, humans are a vital component of real-world problems and they play a major role in shaping other components. Therefore, their perceptions are often necessary to solve problems. However, stakeholders may have different specializations and positions with different levels of knowledge and experience, and their perspectives are generally dominated by their preferences. Dealing with the stakeholders' perceptions/FCMs equally, especially when they are combined into a holistic view, will lead to an inaccurate solution. Thus, FCMs of the stakeholders should be weighted based on suitable criteria that reveal their importance in effectively representing the domain knowledge. To do so, the goal of this paper is to use the fuzzy c-means clustering algorithm in order to obtain different weight values for different stakeholder perspectives (FCMs). These weights are then used to weight the connections in the corresponding FCMs when aggregated into a whole FCM system that reflects an acceptable consensus perception for a given problem. The reason behind the use of this method is that it reveals the extent of convergence and similarity of the views of stakeholders. It first defines a suitable number of clusters. Then, it assigns a memebership degree of each FCM belonging to every defined cluster. Finally, FCMs belonging to the same cluster are as similar as possible, and therefore should take high weights, and vice versa.

To achieve this goal, this paper is structured as follows: Section II overviews FCM approach and presents some related works. The proposed methodology is introduced in Section III, including a brief description of the fuzzy c-means clustering technique. Section IV presents the application of the proposed method on a suitable dataset and discusses the results. Finally, Section V presents the conclusion of the paper.

\section{FCM OVERVIEW AND RELATED WORKS}

FCM approach mimics human cognition of a problem domain (system) without looking at their levels of knowledge. Each human can easily develop their system by exploring uncertain concepts of a given domain and then link these concepts by directed arrows labelled with fuzzy signed values representing cause-effect relationships between concepts, see Fig. 1. The concepts represent the relevant elements of the system such as its variables, factors, attributes, etc. The relationships represent the degree of negative or positive influences between the concepts, where they are encoded into adjacency matrix, Table I.

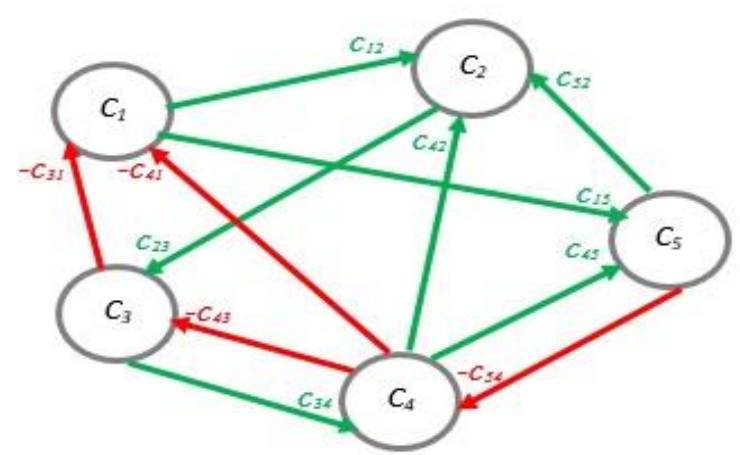

Fig 1. FCM Representing 5 Concepts and the Positive and Negative Relationships between them.

TABLE I. THE ADJACENCY MATRIX OF CONNECTION VALUES OF FCM IN FIG. 1

\begin{tabular}{|c|c|c|c|c|c|}
\hline & $C_{1}$ & $C_{2}$ & $C_{3}$ & $C_{4}$ & $C_{5}$ \\
\hline$C_{1}$ & 0 & $c_{12}$ & 0 & 0 & $c_{15}$ \\
\hline$C_{2}$ & 0 & 0 & $c_{23}$ & 0 & 0 \\
\hline$C_{3}$ & $-c_{31}$ & 0 & 0 & $c_{34}$ & 0 \\
\hline$C_{4}$ & $-c_{41}$ & $c_{42}$ & $-c_{43}$ & 0 & $c_{45}$ \\
\hline$C_{5}$ & 0 & $c_{52}$ & 0 & $-c_{54}$ & 0 \\
\hline
\end{tabular}

FCM approach is a nonlinear dynamic system that allows feedback loops. It uses artificial neural network (ANN) to run the system based on its adjacency matrix and initial state values of concept in order to find the system outcomes, also called FCM inference. The system outcomes represent new state values of concepts and are used to analyse the influence of variables on each other. They are also used to conduct and analyse what-if scenarios in order to explore results that would help in decision making. This process can be applied on each individual FCM system. To address the knowledge of the problem domain as a whole system, the individual FCMs are combined into a collective FCM.

FCM approach has been extensively used in many research domains to model various complex real-world problems. Several studies have considered any relevant stakeholder has an important role in solving such problems [1, 8, 19-21]. The authors in [1] participated different stakeholders to extract their knowledge about environmental problems. They gathered the stakeholders' perspectives of experts and local people as well. Each individual perception was represented in an individual cognitive map which in turn was encoded into adjacency matrix to include the connection values. The authors then combined all perceptions to form a social perception by augmenting each matrix to include all variables identified by all stakeholders. Then, the matrices were added to each other after weighting them using the weighted average method. Each connection value in the resulting combined matrix is the sum of all corresponding values in the individual matrices divided by the number of matrices (FCMs). It is worth noting here that all stakeholders' perceptions have the same weight regardless of their knowledge of the domain. A study in [6] addressed a sustainable socio-economic domain using different knowledge 
resources. The study applied the ordered weighted averaging (OWA) aggregation operators introduced in [22] to aggregate the connection values of FCMs. The aggregation operators are numeric operators such as weighted average, arithmetic mean weighted harmonic averaging, max, min, etc. The above study enhanced OWA method by learning the connection values of relevant stakeholders associated with the operator used for aggregation.

An advance attempt for aggregating different FCMs developed by different stakeholders was in [7]. The above study developed many FCMs from different stakeholders of different groups in solving a complex water scarcity problem. Toward weighting the FCMs before aggregating, the authors used a novel aggregation method to combine FCMs into an overall FCM [7, 23-24]. They assigned a credibility weight for each individual FCM before combining them to each other. The credibility weights of FCMs were calculated based on a novelmeasure, called centrality consensus measure CCM, proposed in [25]. The aforementioned study benefited from the 2-tuple fuzzy linguistic representation model [26] to represent the connection values in the fuzzy $\beta$ values. It is worth mentioning here that this aggregation method can combine fuzzy numerical and linguistic values simultaneously.

Nevertheless, the above studies have not taken into account the consistency and convergence between stakeholders' perspectives (FCMs). When many stakeholders with different knowledge contribute to a given system, this naturally appears inconsistency between them and the holistic system as well. Therefore, the methodology of this paper uses the fuzzy cmeans technique to identify consistent and converging FCMs based on their similarities. Then, it benefits from this process to assign weight values to FCMs. The next section details the methodology proposed in this paper.

\section{Methodology}

Clustering techniques, either hard or soft clustering, have been proven to be effective in grouping similar data into clusters. In hard clustering, the data is grouped into crisp clusters, where each data point either belongs to exactly specific cluster or not, and the clusters cannot overlap, see Fig. 2(A). Unlike the hard clustering, the data point in the soft or fuzzy clustering can belong to multiple clusters with different membership values between 0 and 1 , and the clusters may overlap, see Fig. 2(B).

In this paper, a fuzzy c-means clustering method is used to know the similarities between FCMs by determining how they belong to a defined number of clusters. For each FCM, a memebership degree of its belonging to every cluster will be defined, where FCMs belonging to the same cluster are as similar as possible. The benefit of this is to know the degree of similar FCMs memberships in each cluster. Similarities between FCMs demonstrate the consistency and convergence among the perspectives of FCMs developers. This of course indicates that the more converged and consistent perspectives, the more important they are.
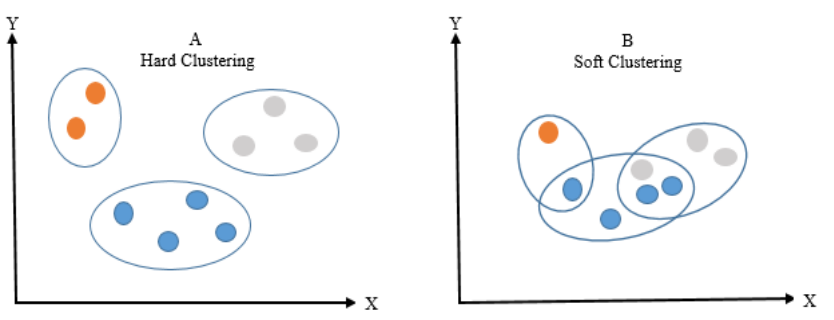

Fig 2. Hard vs Soft Clustering.

Fuzzy c-means, first coined by Dunn [27] and generalized by Bezdek [28, 29], is a fuzzy clustering method in which each element (data point) in a dataset has a degree membership value within the range $[0,1]$ for each cluster. The total of the element membership values for all clusters equals 1. Fuzzy cmeans clustering have been used in many application areas such as clustering, neural network, image analysis, and classification [30].

The structure of the fuzzy c-means algorithm is based on the concept of fuzzy c-partition, proposed by Ruspini [31]. The objective of the fuzzy c-means is to investigate the degree of membership to each sample of data corresponding to each defined cluster centers (centroids). This is done based on the distance between the centroid and the sample of data, in which the degree of membership takes a high value (close to 1) when the sample is near to the centroid. This reflects the similarities of the data samples for clustering.

To put the fuzzy c-means clustering method in the context of the paper methodology, the following steps are performed:

Step1. Prepare the data/samples (FCM matrices) to be an appropriate input for the fuzzy c-means as follows:

a) Each sample (FCM) is represented by $C x \quad C$ adjacency matrix containing connections between concepts, where $C$ is the number of concepts in FCM.

b) Convert the resulting matrix into vector of $C^{2}$ elements. The goal of this conversion process is to create a suitable sample of data for clustering. The data points of the first column in the matrix will be the $[1 \ldots C]$ data points in the new vector, the data points of the second column in the matrix will be $[C+1 \ldots C * 2]$, the elements of the third column in the matrix will be [C*2+1 .. $C * 3]$, and so on, until the data points of the last column in the matrix will be $\left[C^{2}-C+1\right.$.. $\left.C^{2}\right]$.

c) Create a matrix of $N \times C^{2}$, where $N$ is the number of samples (FCMs).

- The output of Stepl is a matrix that includes all connection values between concepts in all FCMs which is then used as data points (samples) input to the fuzzy c-means clustering as shown in Step2.

Step2. Apply the fuzzy c-means algorithm to the data points resulting from Step1 as follows: 
a) Select the number of clusters $(K)$, and the fuzzification parameter $(m), m$ typically in the range $[1.25,2]$.

b) Initialize randomly the membership matrix $U^{0}$ that includes all membership values $u_{i j}$ of belonging of $F C M_{i}$ to clusters $K_{j}$.

c) At iteration $S$, where $S=1$ to number of iterations, calculate centroids $K_{i}$, using the following Equation:

$K_{j}=\frac{\sum_{i=1}^{N} u_{i j}^{m} * X_{i}}{\sum_{i=1}^{N} u_{i j}^{m}}$

where $N$ is the number of samples (FCMs), and $X_{i}$ is the $i^{\text {th }}$ data point.

Calculate the new membership values uij, using the following Equation:

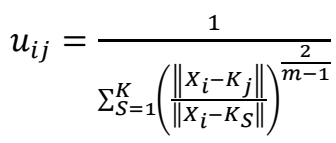

where $\|$ means Euclidian Distance.

a) Update $\mathrm{U}^{\mathrm{S}+1} \leftarrow \mathrm{U}^{\mathrm{S}}$.

b) Repeat steps c to e until a maximum number of iterations is reached or a very small change of the membership values, typically $\mathrm{U}^{\mathrm{S}+1}-\mathrm{U}^{\mathrm{S}}<=0.01$.

- The output of Step2 is a two dimentional matrix; the matrix rows represent the FCMs, the matrix columns represent the clusters, and each row includes the degree membership values of belonging of ith FCM to all clusters, where $\mathrm{i}=1$ to the number of FCMs. This matrix is used to obtain weight values for FCMs as presented in the next Step3.

Step3. Assign weights $(W)$, where $i=1$ to the number of FCMs, to FCM matrices as follows: follows

a) Calculate the importance values of the clusters as

1)For kth cluster, where $\mathrm{k}=1$ to the number of clusters, calculate the total of its degree membership values derived from all FCM matrices.

2)Normalize the calculated values for all clusters to be in the range $[0,1]$. This gives an importance value for each cluster.

b) Assign weight values for FCMs based on the importance values of the clusters as follows:

1) Calculate the importance values for the FCMs as follows:

$i$. For $i^{\text {th }}$ FCM matrix, where $i=1$ to the number of FCMs, then

- For $k^{\text {th }}$ cluster, where $k=1$ to the number of clusters, multiply the degree membership value of belonging of the $i^{\text {th }}$ FCM to the $k^{\text {th }}$ cluster by the importance of the $k^{\text {th }}$ cluster.
- Sum all values of the $i^{\text {th }}$ FCM calculated from step $i$.

2) Use the caculated importance values of FCMs to assign weight values $W$ for these FCMs (matrices).

where $\sum_{i}^{N} W i=1$, and $N$ is the number of FCMs.

Step4. Use the weight values of FCMs in combining the FCMs to obtain consensus, credible and comprehensive FCM system using the following equation:

$F C M=\sum_{i}^{N} W i * F C M i$

where FCM is the group weighted FCM matrix, Wi is the weight of ith FCM matrix.

Next section demonstrates the application of our proposed method on a real dataset.

\section{Testing the Proposed Method}

The proposed method was tested using the dataset collected in the study [23]. The dataset included 35 samples (FCMs) developed from 35 different stakeholders representing a major real world problem "water scarcity problem". Each FCM contained 13 same concepts. Thus, the adjacency matrix of each sample was $13 \times 13$ dimensions and included the connection values between these concepts.

Based on Step 1.b in the proposed methodology, a vector of $1 \times 13^{2}$ was created for each adjacency matrix of each FCM. Then, the input matrix for fuzzy c-means clustering was of $35 \times 169$ and included all connection values between concepts in all FCMs, (Step 1.c in the proposed methodology). We used the silhouette criteria introduce in [32] to select the suitable number of clusters and centroids, accordingly. This number was 10 cluster. After that, the steps of the fuzzy cmeans clustering algorithm (Step2.b-f in the proposed methodology) were implemented to calculate the final membership values $u_{i j}$ of belonging of FCMs to all clusters. The outcomes (membership values) of the above steps are shown in Table II.

Then, we calculated the importance of the clusters according to the Step3.a in the proposed methodology. Table III shows these importance values. We then used Step3.b to find the importance values of FCMs by multiplying the membership values of their belongings to the cluster by calculated importance values of the clusters, see Table IV.

Finally, we utilized the calculated importance values of FCMs to assign weight values for these FCMs. This process was performed using a ratio method, which was applied to the imprtance values to make the resulting weight values within the range $[0,1]$, and the total of these weight values equal to 1 . The last column in the Table IV shows the weights assigned to the FCMs. 
TABle II. The Degree Membership VAlues, $U_{\mathrm{J}}$, OF Belonging of FCMs to All Clusters

\begin{tabular}{|c|c|c|c|c|c|c|c|c|c|c|}
\hline \begin{tabular}{|l} 
Cluster ID \\
FCM ID
\end{tabular} & $\begin{array}{c}\text { Cluster } \\
1\end{array}$ & $\begin{array}{c}\text { Cluster } \\
2\end{array}$ & $\begin{array}{c}\text { Cluster } \\
3\end{array}$ & $\begin{array}{c}\text { Cluster } \\
4\end{array}$ & $\begin{array}{c}\text { Cluster } \\
5\end{array}$ & $\begin{array}{c}\text { Cluster } \\
6\end{array}$ & $\begin{array}{c}\text { Cluster } \\
7\end{array}$ & $\begin{array}{c}\text { Cluster } \\
8\end{array}$ & $\begin{array}{c}\text { Cluster } \\
9\end{array}$ & $\begin{array}{r}\text { Cluster } \\
10\end{array}$ \\
\hline$F C M 1$ & 0.01532 & 0.00009 & 0.98080 & 0.00001 & 0.00004 & 0.00000 & 0.00109 & 0.00038 & 0.00008 & 0.00218 \\
\hline FCM 2 & 0.00020 & 0.99077 & 0.00073 & 0.00027 & 0.00016 & 0.00015 & 0.00065 & 0.00135 & 0.00064 & 0.00508 \\
\hline$F C M 3$ & 0.00504 & 0.00079 & 0.99114 & 0.00002 & 0.00006 & 0.00001 & 0.00032 & 0.00030 & 0.00004 & 0.00229 \\
\hline$F C M 4$ & 0.00020 & 0.00039 & 0.99905 & 0.00000 & 0.00000 & 0.00000 & 0.00015 & 0.00001 & 0.00003 & 0.00016 \\
\hline FCM 5 & 0.00012 & 0.99312 & 0.00032 & 0.00010 & 0.00005 & 0.00007 & 0.00481 & 0.00058 & 0.00020 & 0.00063 \\
\hline$F C M 6$ & 0.00048 & 0.99387 & 0.00095 & 0.00005 & 0.00002 & 0.00001 & 0.00062 & 0.00107 & 0.00061 & 0.00231 \\
\hline$F C M 7$ & 0.00002 & 0.00002 & 0.00000 & 0.99964 & 0.00000 & 0.00000 & 0.00004 & 0.00021 & 0.00000 & 0.00007 \\
\hline$F C M 8$ & 0.00014 & 0.00006 & 0.00047 & 0.00000 & 0.00004 & 0.00001 & 0.00007 & 0.00016 & 0.99874 & 0.00030 \\
\hline FCM 9 & 0.00027 & 0.00059 & 0.00006 & 0.00001 & 0.00002 & 0.00000 & 0.00012 & 0.00010 & 0.99859 & 0.00023 \\
\hline FCM 10 & 0.00005 & 0.00083 & 0.00006 & 0.00001 & 0.00001 & 0.00002 & 0.00012 & 0.00002 & 0.99858 & 0.00029 \\
\hline FCM 11 & 0.00006 & 0.00024 & 0.00011 & 0.00003 & 0.00006 & 0.00001 & 0.00038 & 0.00035 & 0.00008 & 0.99869 \\
\hline FCM 12 & 0.00346 & 0.00063 & 0.00409 & 0.00013 & 0.00006 & 0.00001 & 0.00268 & 0.00240 & 0.00010 & 0.98644 \\
\hline FCM 13 & 0.00079 & 0.00369 & 0.00225 & 0.00036 & 0.00002 & 0.00002 & 0.00640 & 0.00136 & 0.00016 & 0.98495 \\
\hline FCM 14 & 0.00035 & 0.00010 & 0.00008 & 0.00001 & 0.00002 & 0.00000 & 0.00033 & 0.00016 & 0.00001 & 0.99896 \\
\hline FCM 15 & 0.00087 & 0.00150 & 0.00075 & 0.00004 & 0.00002 & 0.00002 & 0.00153 & 0.00059 & 0.00010 & 0.99458 \\
\hline$F C M 16$ & 0.01067 & 0.00328 & 0.00119 & 0.00085 & 0.00377 & 0.00025 & 0.00711 & 0.00355 & 0.00139 & 0.96794 \\
\hline FCM 17 & 0.99510 & 0.00009 & 0.00052 & 0.00001 & 0.00002 & 0.00000 & 0.00240 & 0.00039 & 0.00002 & 0.00145 \\
\hline FCM 18 & 0.99928 & 0.00001 & 0.00026 & 0.00000 & 0.00001 & 0.00000 & 0.00023 & 0.00003 & 0.00000 & 0.00018 \\
\hline FCM 19 & 0.00117 & 0.00006 & 0.00021 & 0.00003 & 0.00004 & 0.00000 & 0.99758 & 0.00030 & 0.00000 & 0.00060 \\
\hline FCM 20 & 0.00028 & 0.00066 & 0.00009 & 0.00027 & 0.00003 & 0.00000 & 0.00101 & 0.99726 & 0.00001 & 0.00039 \\
\hline FCM 21 & 0.00001 & 0.00001 & 0.00000 & 0.99984 & 0.00000 & 0.00000 & 0.00004 & 0.00004 & 0.00000 & 0.00005 \\
\hline FCM 22 & 0.00005 & 0.00005 & 0.00004 & 0.00001 & 0.99903 & 0.00005 & 0.00036 & 0.00035 & 0.00001 & 0.00006 \\
\hline FCM 23 & 0.00003 & 0.00005 & 0.00002 & 0.00000 & 0.00000 & 0.00000 & 0.99965 & 0.00013 & 0.00000 & 0.00013 \\
\hline FCM 24 & 0.00031 & 0.00035 & 0.00013 & 0.00004 & 0.00167 & 0.00001 & 0.99447 & 0.00164 & 0.00018 & 0.00121 \\
\hline FCM 25 & 0.00314 & 0.00127 & 0.00231 & 0.00019 & 0.97391 & 0.00026 & 0.00779 & 0.00260 & 0.00285 & 0.00569 \\
\hline FCM 26 & 0.00006 & 0.00027 & 0.00006 & 0.00012 & 0.00017 & 0.00001 & 0.00205 & 0.99595 & 0.00005 & 0.00124 \\
\hline$F C M 27$ & 0.00108 & 0.00012 & 0.00012 & 0.00003 & 0.99677 & 0.00015 & 0.00071 & 0.00037 & 0.00004 & 0.00062 \\
\hline FCM 28 & 0.99897 & 0.00001 & 0.00085 & 0.00000 & 0.00001 & 0.00000 & 0.00003 & 0.00001 & 0.00001 & 0.00011 \\
\hline FCM 29 & 0.00000 & 0.00000 & 0.00000 & 0.00000 & 0.00000 & 0.99999 & 0.00000 & 0.00000 & 0.00000 & 0.00000 \\
\hline FCM 30 & 0.00269 & 0.00075 & 0.99019 & 0.00003 & 0.00009 & 0.00003 & 0.00252 & 0.00194 & 0.00076 & 0.00100 \\
\hline FCM 31 & 0.00000 & 0.00000 & 0.00000 & 0.00000 & 0.00001 & 0.99998 & 0.00000 & 0.00000 & 0.00000 & 0.00000 \\
\hline FCM 32 & 0.00021 & 0.99403 & 0.00063 & 0.00001 & 0.00008 & 0.00008 & 0.00142 & 0.00148 & 0.00030 & 0.00176 \\
\hline FCM 33 & 0.00021 & 0.00026 & 0.00023 & 0.00006 & 0.00010 & 0.00004 & 0.00150 & 0.99659 & 0.00001 & 0.00100 \\
\hline FCM 34 & 0.00024 & 0.00075 & 0.00021 & 0.00003 & 0.00002 & 0.00001 & 0.99577 & 0.00167 & 0.00002 & 0.00128 \\
\hline FCM 35 & 0.00001 & 0.00001 & 0.00000 & 0.00000 & 0.99990 & 0.00000 & 0.00002 & 0.00003 & 0.00000 & 0.00002 \\
\hline
\end{tabular}

TABLE III. THE IMPORTANCE VALUES OF THE Clusters

\begin{tabular}{|l|l|l|l|l|l|l|l|l|l|l|}
\hline Cluster ID & 1 & 2 & 3 & 4 & 5 & 6 & 7 & 8 & 9 \\
\hline $\begin{array}{l}\text { Cluster } \\
\text { importance }\end{array}$ & 0.30408 & 0.39887 & 0.39779 & 0.20022 & 0.39762 & 0.20012 & 0.40340 & 0.30134 & 0.30036 & 0.59619 \\
\hline
\end{tabular}


TABLE IV. FCMS IMPORTANCE VALUES AND THEIR CALCULATED WEIGHT VALUES, ACCORdINGLY

\begin{tabular}{|c|c|c|c|c|c|c|c|c|c|c|c|c|}
\hline $\begin{array}{l}\text { Cluster ID } \\
\text { FCM ID }\end{array}$ & $\begin{array}{l}\text { Cluster } \\
1\end{array}$ & $\begin{array}{l}\text { Cluster } \\
2\end{array}$ & $\begin{array}{l}\text { Cluster } \\
3\end{array}$ & $\begin{array}{l}\text { Cluster } \\
4\end{array}$ & $\begin{array}{l}\text { Cluster } \\
5\end{array}$ & $\begin{array}{l}\text { Cluster } \\
6\end{array}$ & $\begin{array}{l}\text { Cluster } \\
7\end{array}$ & $\begin{array}{l}\text { Cluster } \\
8\end{array}$ & $\begin{array}{l}\text { Cluster } \\
9\end{array}$ & $\begin{array}{l}\text { Cluster } \\
10\end{array}$ & $\begin{array}{l}\text { FCM } \\
\text { Importance }\end{array}$ & FCM Weight \\
\hline$F C M 1$ & 0.00466 & 0.00004 & 0.39015 & 0.00000 & 0.00002 & 0.00000 & 0.00044 & 0.00012 & 0.00002 & 0.00130 & 0.39675 & 0.02945 \\
\hline$F C M 2$ & 0.00006 & 0.39519 & 0.00029 & 0.00005 & 0.00006 & 0.00003 & 0.00026 & 0.00041 & 0.00019 & 0.00303 & 0.39958 & 0.02966 \\
\hline$F C M 3$ & 0.00153 & 0.00032 & 0.39427 & 0.00000 & 0.00002 & 0.00000 & 0.00013 & 0.00009 & 0.00001 & 0.00137 & 0.39774 & 0.02952 \\
\hline FCM 4 & 0.00006 & 0.00016 & 0.39741 & 0.00000 & 0.00000 & 0.00000 & 0.00006 & 0.00000 & 0.00001 & 0.00010 & 0.39780 & 0.02953 \\
\hline FCM 5 & 0.00004 & 0.39613 & 0.00013 & 0.00002 & 0.00002 & 0.00001 & 0.00194 & 0.00017 & 0.00006 & 0.00038 & 0.39890 & 0.02961 \\
\hline FCM 6 & 0.00015 & 0.39643 & 0.00038 & 0.00001 & 0.00001 & 0.00000 & 0.00025 & 0.00032 & 0.00018 & 0.00137 & 0.39910 & 0.02962 \\
\hline$F C M 7$ & 0.00001 & 0.00001 & 0.00000 & 0.20015 & 0.00000 & 0.00000 & 0.00002 & 0.00006 & 0.00000 & 0.00004 & 0.20028 & 0.01487 \\
\hline FCM 8 & 0.00004 & 0.00003 & 0.00019 & 0.00000 & 0.00002 & 0.00000 & 0.00003 & 0.00005 & 0.29999 & 0.00018 & 0.30051 & 0.02231 \\
\hline FCM 9 & 0.00008 & 0.00024 & 0.00002 & 0.00000 & 0.00001 & 0.00000 & 0.00005 & 0.00003 & 0.29994 & 0.00014 & 0.30051 & 0.02231 \\
\hline FCM 10 & 0.00002 & 0.00033 & 0.00003 & 0.00000 & 0.00001 & 0.00000 & 0.00005 & 0.00001 & 0.29994 & 0.00017 & 0.30055 & 0.02231 \\
\hline FCM 11 & 0.00002 & 0.00009 & 0.00004 & 0.00001 & 0.00002 & 0.00000 & 0.00015 & 0.00011 & 0.00002 & 0.59541 & 0.59588 & 0.04423 \\
\hline FCM 12 & 0.00105 & 0.00025 & 0.00163 & 0.00003 & 0.00003 & 0.00000 & 0.00108 & 0.00072 & 0.00003 & 0.58810 & 0.59292 & 0.04401 \\
\hline FCM 13 & 0.00024 & 0.00147 & 0.00089 & 0.00007 & 0.00001 & 0.00000 & 0.00258 & 0.00041 & 0.00005 & 0.58721 & 0.59295 & 0.04401 \\
\hline FCM 14 & 0.00011 & 0.00004 & 0.00003 & 0.00000 & 0.00001 & 0.00000 & 0.00013 & 0.00005 & 0.00000 & 0.59557 & 0.59593 & 0.04423 \\
\hline FCM 15 & 0.00026 & 0.00060 & 0.00030 & 0.00001 & 0.00001 & 0.00000 & 0.00062 & 0.00018 & 0.00003 & 0.59295 & 0.59496 & 0.04416 \\
\hline FCM 16 & 0.00325 & 0.00131 & 0.00047 & 0.00017 & 0.00150 & 0.00005 & 0.00287 & 0.00107 & 0.00042 & 0.57708 & 0.58818 & 0.04366 \\
\hline FCM 17 & 0.30260 & 0.00003 & 0.00021 & 0.00000 & 0.00001 & 0.00000 & 0.00097 & 0.00012 & 0.00000 & 0.00087 & 0.30480 & 0.02262 \\
\hline FCM 18 & 0.30387 & 0.00000 & 0.00010 & 0.00000 & 0.00000 & 0.00000 & 0.00009 & 0.00001 & 0.00000 & 0.00011 & 0.30419 & 0.02258 \\
\hline FCM 19 & 0.00036 & 0.00002 & 0.00008 & 0.00001 & 0.00002 & 0.00000 & 0.40242 & 0.00009 & 0.00000 & 0.00036 & 0.40336 & 0.02994 \\
\hline FCM 20 & 0.00008 & 0.00026 & 0.00004 & 0.00005 & 0.00001 & 0.00000 & 0.00041 & 0.30051 & 0.00000 & 0.00023 & 0.30161 & 0.02239 \\
\hline$F C M 21$ & 0.00000 & 0.00001 & 0.00000 & 0.20019 & 0.00000 & 0.00000 & 0.00001 & 0.00001 & 0.00000 & 0.00003 & 0.20026 & 0.01486 \\
\hline$F C M 22$ & 0.00001 & 0.00002 & 0.00002 & 0.00000 & 0.39724 & 0.00001 & 0.00015 & 0.00011 & 0.00000 & 0.00004 & 0.39759 & 0.02951 \\
\hline$F C M 23$ & 0.00001 & 0.00002 & 0.00001 & 0.00000 & 0.00000 & 0.00000 & 0.40326 & 0.00004 & 0.00000 & 0.00008 & 0.40341 & 0.02994 \\
\hline$F C M 24$ & 0.00009 & 0.00014 & 0.00005 & 0.00001 & 0.00066 & 0.00000 & 0.40117 & 0.00049 & 0.00005 & 0.00072 & 0.40339 & 0.02994 \\
\hline$F C M 25$ & 0.00096 & 0.00050 & 0.00092 & 0.00004 & 0.38725 & 0.00005 & 0.00314 & 0.00078 & 0.00086 & 0.00339 & 0.39789 & 0.02953 \\
\hline$F C M 26$ & 0.00002 & 0.00011 & 0.00002 & 0.00002 & 0.00007 & 0.00000 & 0.00083 & 0.30012 & 0.00002 & 0.00074 & 0.30195 & 0.02241 \\
\hline FCM 27 & 0.00033 & 0.00005 & 0.00005 & 0.00001 & 0.39634 & 0.00003 & 0.00029 & 0.00011 & 0.00001 & 0.00037 & 0.39757 & 0.02951 \\
\hline$F C M 28$ & 0.30377 & 0.00000 & 0.00034 & 0.00000 & 0.00000 & 0.00000 & 0.00001 & 0.00000 & 0.00000 & 0.00006 & 0.30420 & 0.02258 \\
\hline FCM 29 & 0.00000 & 0.00000 & 0.00000 & 0.00000 & 0.00000 & 0.20012 & 0.00000 & 0.00000 & 0.00000 & 0.00000 & 0.20012 & 0.01485 \\
\hline FCM 30 & 0.00082 & 0.00030 & 0.39389 & 0.00001 & 0.00004 & 0.00001 & 0.00102 & 0.00058 & 0.00023 & 0.00059 & 0.39748 & 0.02950 \\
\hline FCM 31 & 0.00000 & 0.00000 & 0.00000 & 0.00000 & 0.00000 & 0.20012 & 0.00000 & 0.00000 & 0.00000 & 0.00000 & 0.20013 & 0.01485 \\
\hline FCM 32 & 0.00006 & 0.39649 & 0.00025 & 0.00000 & 0.00003 & 0.00002 & 0.00057 & 0.00044 & 0.00009 & 0.00105 & 0.39901 & 0.02962 \\
\hline$F C M 33$ & 0.00006 & 0.00011 & 0.00009 & 0.00001 & 0.00004 & 0.00001 & 0.00061 & 0.30031 & 0.00000 & 0.00059 & 0.30183 & 0.02240 \\
\hline$F C M 34$ & 0.00007 & 0.00030 & 0.00008 & 0.00001 & 0.00001 & 0.00000 & 0.40169 & 0.00050 & 0.00001 & 0.00076 & 0.40344 & 0.02995 \\
\hline FCM 35 & 0.00000 & 0.00000 & 0.00000 & 0.00000 & 0.39758 & 0.00000 & 0.00001 & 0.00001 & 0.00000 & 0.00001 & 0.39762 & 0.02951 \\
\hline
\end{tabular}

As shown in Table IV, the weight value of both FCM 14 and FCM 11, (0.04423), was the highest of the weight values. This means that FCM 14 and FCM 11 are the most important FCMs which are well perceive the domain knowledge of the problem and that their developers are the most credible stakeholders. Thus, when multiplying the matrices of these FCMs by their credible weights during combining them to other FCMs (Step4 in the methodology), this will enhance the compatibility and reliability of the resulting comprehensive FCM system.

On the other hand and as shwon in Table IV, the weight value of both FCM 29 and FCM 31, (0.01485), was the lowest of FCMs weight values. This indicates that these FCMs are less important in representing domain knowledge, and therefore, the stakeholders who developed these FCMs are less reliable (less experienced or knowledgeable). Therefore, the matrices of these FCMs should be weighted with lower weights 
in the combination process. Other FCMs with different weight values are treated with the same steps above. It should be noted here that any participation of all relevant stakeholders should not be neglected as mentioned above, but that their different levels of knowledge and preferences should be taken into consideration in any further process.

\section{CONCLUSION}

This paper introduces a new method to assign weights for fuzzy cognitive maps (FCMs) developed by different stakeholders representing a given complex problem domain. The main goal of this is to use these weight values in order to reach an accurate consensus representation of the problem after combining the weighted FCMs. To achieve this, important FCMs are given high weights, and vice versa. This paper uses fuzzy c-means clustering algorithm to reveal the importance of FCMs. By this algorithm, the membership values of belonging of FCMs to the clusters are calculated. The membership values are computed based on the similarities between FCMs. Based on this, the importance values of cluster were extracted. Then, importance values of FCMs are identified based on their contributions to the clusters taken into accounts the importance values of the clusters. Consequently, weight values are assigned to FCMs using FCMs importance values. Finally, experimenting with the proposed method on a real dataset has proved its suitability and efficiency in assigning credible weights of for FCMs. As a future work, we intend to combine this proposed method with other effective techniques for more reliable weights either for FCMs or the connections in the FCMs.

\section{REFERENCES}

[1] Ozesmi, U. and S. Ozesmi, Ecological models based on people's knowledge: a multi-step fuzzy cognitive mapping approach. Ecological Modelling, 2004. 176(1-2): p. 43-64.

[2] Zadeh, L.A., Fuzzy logic, neural networks, and soft computing. Acm Transactions on Information Systems, 1994. 37 p. 77 - 84.

[3] Virapongse, A., et al., A social-ecological systems approach for environmental management. Journal of Environmental Management, 2016. 178: p. 83-91.

[4] Stojanovic, T., et al., The "social" aspect of social-ecological systems a critique of analytical frameworks and findings from a multisite study of coastal sustainability. Ecology and Society, 2016. 21(3).

[5] Gray, S., et al., Modeling the integration of stakeholder knowledge in social-ecological decision-making: Benefits and limitations to knowledge diversity. Ecological Modelling, 2012. 229(0): p. 88-96.

[6] Papageorgiou, K., et al., Fuzzy Cognitive Map-Based Sustainable SocioEconomic Development Planning for Rural Communities. Sustainability, 2019. 12(1).

[7] Obiedat, M. and S. Samarasinghe, A novel semi-quantitative Fuzzy Cognitive Map model for complex systems for addressing challenging participatory real life problems. Applied Soft Computing, 2016. 48: p. 91-110.

[8] Ozesmi, U. and S. Ozesmi, A participatory approach to ecosystem conservation: Fuzzy cognitive maps and stakeholder group analysis in Uluabat Lake, Turkey. Environmental Management, 2003. 31(4): p. 518-531.

[9] Gray, S., et al., Using fuzzy cognitive mapping as a participatory approach to analyze change, preferred states, and perceived resilience of social-ecological systems. Ecology and Society, 2015. 20.

[10] Axelrod, R., Structure of Decision: The Cognitive Maps of Political Elites. 1976, Englewood Cliffs, New Jersy: Prentice-Hall.
[11] Zadeh, L.A., Fuzzy Sets*. Information and Control 1965. 8(3): p. 338353

[12] Ross, T.J., Fuzzy Logic with Engineering Applications 2004: John Wiley \& Sons, Ltd.

[13] Kosko, B., Fuzzy cognitive maps. International Journal of Man-Machine Studies, 1986. 24(1): p. 65-75.

[14] Mobolurin, A., Generating consensus fuzzy cognitive maps, in Proceedings of the 1997 IASTED International Conference on Intelligent Information Systems (IIS '97). 1997, IEEE Computer Society.

[15] Nair, A., D. Reckien, and M.F.A.M. Maarseveen, A generalised fuzzy cognitive mapping approach for modelling complex systems. Applied Soft Computing, 2019. 84(3): p. 1-13.

[16] Tan, C., H. Teo, and K. Wei, Promoting consensus in small decision making groups. Information \& Management, 1995. 28(4): p. 251-259.

[17] Papageorgiou, E.I., A.T. Markinos, and T.A. Gemtos, Soft Computing Technique of Fuzzy Cognitive Maps to Connect Yield Defining Parameters with Yield in Cotton Crop Production in Central Greece as a Basis for a Decision Support System for Precision Agriculture Application, in Fuzzy Cognitive Maps. 2010, Springer Berlin Heidelberg. p. 325-362.

[18] Kumar, A. and G. Karmakar, Aggregation of opinions using fuzzy numbers. International Journal of Systems Science, 2001. 32(12): p. 1399-1411.

[19] Mehryar, S., et al. Making Use of Fuzzy Cognitive Maps in AgentBased Modeling. in Advances in Social Simulation. 2020. Cham: Springer International Publishing.

[20] Wu, W., et al., Including stakeholder input in formulating and solving real-world optimisation problems: Generic framework and case study. Environmental Modelling \& Software, 2016. 79: p. 197-213.

[21] Gregory, A.J., et al., Stakeholder identification and engagement in problem structuring interventions. European Journal of Operational Research, 2020. 283(1): p. 321-340.

[22] Yager, R.R., On ordered weighted averaging aggregation operators in multicriteria decision-making. Ieee Transactions on Systems Man and Cybernetics, 1988. 18(1): p. 183-190.

[23] Obiedat, M., Robust semi-quantitative fuzzy cognitive map model for complex systems and case study: mitigating the water scarcity problem in Joran, in Department of Environmental Management. 2013, Lincoln University: Lincoln University. p. 277.

[24] Obiedat, M. and S. Samarasinghe, Fuzzy Representation and Aggregation of Fuzzy Cognitive Maps, in 20th International Congress on Modelling and Simulation. 2013: Adelaide, Australia.

[25] Obiedat, M., S. Samarasinghe, and G. Strickert, A New Method for Identifying the Central Nodes in Fuzzy Cognitive Maps using Consensus Centrality Measure, in 19th International Congress on Modelling and Simulation. 2011: Perth, Australia.

[26] Herrera, F. and L. Martinez, A 2-tuple fuzzy linguistic representation model for computing with words. Ieee Transactions on Fuzzy Systems, 2000. 8(6): p. 746-752.

[27] Dunn, J.C., A Fuzzy Relative of the ISODATA Process and Its Use in Detecting Compact Well-Separated Clusters. Journal of Cybernetics, 1973. 3(3): p. 32-57.

[28] Bezdek, J.C., Pattern Recognition with Fuzzy Objective Function Algorithms. 1981: Kluwer Academic Publishers.

[29] Hathaway, R.J. and J.C. Bezdek, Recent convergence results for the fuzzy c-means clustering algorithms. Journal of Classification, 1988. $5(2)$ : p. $237-247$.

[30] Nayak, J., B. Naik, and D.H. Behera, Fuzzy C-Means (FCM) Clustering Algorithm: A Decade Review from 2000 to 2014. 2015. p. 133-149.

[31] Ruspini, E.H., A new approach to clustering. Information and Control, 1969. 15(1): p. 22-32.

[32] Rousseeuw, P.J., Silhouettes: A graphical aid to the interpretation and validation of cluster analysis. Journal of Computational and Applied Mathematics, 1987. 20: p. 53-65. 